Research Paper

\title{
Pulmonary Vein Isolation in 2012: Is It Necessary to Perform a Time Consuming Electrophysical Mapping or Should We Focus on Rapid and Safe Therapies? A Retrospective Analysis of Different Ablation Tools
}

\author{
Petra Maagh ${ }^{\bowtie}$, Thomas Butz², Gunnar Plehn², Arndt Christoph¹, Axel Meissner ${ }^{1}$ \\ 1. Department of Cardiology and Angiology, Klinikum Merheim, University Witten/Herdecke/Germany, Ostmerheimer Str. 200, 51109 \\ Cologne, Germany; \\ 2. Department of Cardiology and Angiology, Ruhr-University Bochum/Germany, Hölkeskampring 40, 45625 Herne, Germany.
}

\begin{abstract}
$\square$ Corresponding author: Petra Maagh, Klinikum Merheim, University Witten/Herdecke/Germany, Ostmerheimer Str. 200, 51109 Cologne, Germany. Tel.: 0049/221 8907-3457 Fax: 0049/221 8907-3488 e-mail: Petra.Maagh@rub.de.
\end{abstract}

(ㅇ Ivyspring International Publisher. This is an open-access article distributed under the terms of the Creative Commons License (http://creativecommons.org/ licenses/by-nc-nd/3.0/). Reproduction is permitted for personal, noncommercial use, provided that the article is in whole, unmodified, and properly cited.

Received: 2012.06.21; Accepted: 2012.10.15; Published: 2012.12.07

\begin{abstract}
Background: Pulmonary Vein Isolation (PVI) is evolving as an established treatment option in atrial fibrillation (AF). Different fluoroscopy-guided ablation devices exist either on the basis of expandable circumferential and mesh designs with mapping and ablation of pulmonary vein potentials, or of a balloon technology, a "single shot" device with a purely anatomical approach. Systematic comparisons between procedure duration (PD), fluoroscopy time (FT) and clinical outcome in using different ablation tools are lacking in the literature.

Methods: In a single center retrospective analysis, II $9 \mathrm{PVI}$ procedures were performed between August 2008 and March $20 \mathrm{II}$ in paroxysmal AF (PAF, 59.7\%) and persistent AF (persAF, $40.3 \%$ ) patients with mean age of $59.4 \pm 10.3$ years and history of AF since $8.1 \pm 9.7$ months. The PVI procedures were evaluated by comparing PD and FT using I) the High Density Mesh Mapper (HDMM), II) the High Density Mesh Ablator (HDMA), and III) the Arctic Front ${ }^{\circledR}$ Cryoballoon. The primary endpoints were FT and PD, the secondary endpoint was procedural safety and efficacy in short- and longterm follow-up.

Results: The procedures performed for II 9 patients $(63.0 \%$ male) included $42 \mathrm{PVIs}$ with the HDMM (35.3 \%), 47 with the HDMA (39.5\%) and 30 with the cryoballoon (25.2 \%). Comparing the 30 first procedures in groups of 10 in the HDMM and HDMA group, PD and FT fell in the HDMM group (PD from 257.5 to $220.9 \mathrm{~min}$ and FT from 80.5 to $67.3 \mathrm{~min}$, both $\mathrm{P}<$ 0.05 ) as well as in the HDMA group (PD from I82.9 to I47.2 min and FT from 4I.02 to 29.I min, both $p<0.05)$. In the cryoballoon group, there was a steep learning curve with a steady state after the first 10 procedures (PD and FT decreased significantly from 189.5 to $138.1 \mathrm{~min}$ and 36.9 to $27.3 \mathrm{~min}, \mathrm{p}$ values 0.005 and 0.05 respectively). With respect to recurrence of $\mathrm{AF}$ in a 24 months follow up, the HDMM and cryoballoon group showed comparable results with $\sim 72 \%$ of patients free of arrhythmias. None of the patients died due to severe complications, or suffered a hemodynamic relevant pericardial effusion and/or stroke. Impairment of the phrenic nerve was observed in three patients.

Conclusion: Use of the cryoballoon technology was associated with a steep learning curve and a reduced PD and FT; the long-term outcome was similar compared with the HDMM group. The efficacy and safety of the devices but also PD and FT should be respected as the strongest indicators of the quality of ablation. Further studies with long time follow-ups will show if the
\end{abstract}


time for correct mapping of the PV potentials is a price we should be willing to pay or if we should adopt a "wait-and-see" attitude referring the AF recurrence.

Key words: Atrial fibrillation; different ablation tools; procedure duration, fluoroscopy time, safety.

\section{Introduction}

In the past 10 years, catheter ablation for the treatment of patients with symptomatic atrial fibrillation (AF) has evolved from a highly investigational technique to its current role as a curative early treatment of AF [1]. The generally accepted endpoint is the complete electrical PV isolation with demonstration of block between the left atrium (LA) and the PVs [2]. The procedure can be performed with reasonable efficacy and safety by experienced operators. The fluoroscopy-guided ablation procedures involve radiation burden, particularly in patients with subsequent ablation procedures in case of recurrences of AF. Great interest exists in the development of new tools that will improve the efficacy and safety of AF ablation but also shorten the procedure duration (PD) and fluoroscopy time (FT) [3]. Furthermore, the new tools should allow ablation to be performed by operators with little prior experience. Studies reporting in comparative manner learning curves, PD, FT and associated complications using different ablation techniques are lacking. In our single center study, we aim to compare three approaches (in the following I-III) to perform PVI:

- The High density Mesh Mapper (HDMM, BARD Electrophysiology, Lowell, MA, USA) as already described by our group [4]: segmental ostial ablation was performed by irrigated radiofrequency (RF) application around the HDMM requiring transseptal puncture (TSP) twice, one for the HDMM itself as the mapping catheter, the second one for the ablation. Entry- and exit conduction block, as well as decreased local electrode amplitude, were endpoints for short-term successful ablation.

- The High Density Mesh Ablator (HDMA, BARD Electrophysiology, Lowell, MA, USA) as already described by our group [5]: complete elimination or disconnection of PV signals was performed with a catheter combining direct RF energy delivery and high-density circumferential mapping in a single unit.

- Arctic Front ${ }^{\circledR}$ (Medtronic Cryocath ${ }^{\circledR}$, Chemin Stemarie Kirkland, QC, Canada), an anatomically based approach without mapping capacities using a cryoballoon ablation system that al- lows cooling the temperature for circumferential ablation $[6,7,8]$. The endpoint was the PVI checked by a circular mapping catheter.

\section{Methods}

\section{Study design}

In our single center, we designed a retrospective analysis between 2008 and 2011 to compare the initial experiences in fluoroscopy-guided approaches for PVI regarding the PD and FT using I) the HDMM with irrigated-tip RF for segmental ostial ablation, II) the single device RF and mapping technique of the HDMA, and III) the single device cryothermal energy of the Arctic Front ${ }^{\circledR}$ cryoballoon. For PVI, we started recording $\mathrm{PD}$ and FT with the transfemoral puncture and stopped recording the time when disconnection of all PVs was achieved. No intraprocedural imaging except for fluoroscopy was used in this study. The primary endpoints were FT and PD, the secondary endpoint was procedural safety and efficacy in shortand long-term follow-up.

In our centre, there were two operators working with all three ablation systems. One of them had gone through a learning curve prior to enrolling patients; the second was early in his learning curve. Procedures parameters ( $\mathrm{PD}, \mathrm{FT}$, and acute procedure outcome) were routinely recorded in the laboratory log.

\section{Patients selected and procedure management}

This retrospective analysis enrolled 119 patients with symptomatic drug refractory paroxysmal AF (PAF) and persistent AF (persAF) undergoing circumferential LA PVI. Table 1 shows the baseline characteristics of the patients. All patients underwent transthoracic echocardiography to determine the diameter of the LA. To assess PV size and geometry, magnetic resonance imaging (MRI) or computed tomography images were obtained before intervention. All procedures were performed under conscious sedation and analgesia with appropriate doses of midazolam and fentanyl. After TSP, heparin was maintained to achieve an activated clotting time $>300 \mathrm{~s}$. Measurements were performed every $30 \mathrm{~min}$. 
Table I: Baseline patient characteristics.

\begin{tabular}{|c|c|c|c|}
\hline & HDMM & HDMA & Cryoballoon \\
\hline Number & 42 & 47 & 30 \\
\hline Male & $29(69.0 \%)$ & $27(57.4 \%)$ & $19(63.3 \%)$ \\
\hline Age (years) & $60.6 \pm 10.4$ & $57.9 \pm 10.3$ & $59.9 \pm 10.3$ \\
\hline Length (cm) & $177.4 \pm 9.3$ & $170.2 \pm 23.4$ & $160.2 \pm 34.4$ \\
\hline Weight (kg) & $85.2 \pm 13.9$ & $96.3 \pm 30.5$ & $106.1 \pm 41.3$ \\
\hline BMI & $26.9 \pm 4.3$ & & \\
\hline \multicolumn{4}{|l|}{ AF type } \\
\hline paroxysmal & $27(64.3 \%)$ & $23(48.9 \%)$ & $21(70.0 \%)$ \\
\hline persistent & $15(35.7 \%)$ & $24(51.1 \%)$ & $9(30.0 \%)$ \\
\hline AF history (months) & $7.7 \pm 5.3$ & $5.6 \pm 3.5$ & $12.5 \pm 17.3$ \\
\hline Prior hospitalization & $5.6 \pm 4.7$ & $6.6 \pm 3.3$ & $3.2 \pm 1.3$ \\
\hline $\mathbf{A A D s}^{\mathbf{a}}$ & $42(100.0 \%)$ & $47(100.0 \%)$ & $30(100 \%)$ \\
\hline \multicolumn{4}{|l|}{ Prior PVI } \\
\hline without & $38(90.5 \%)$ & $34(72.3 \%)$ & $18(60.0 \%)$ \\
\hline once & $3(7.1 \%)$ & $12(25.5 \%)$ & $11(36.7 \%)$ \\
\hline twice & $1(2.4 \%)$ & $1(2.1 \%)$ & $1(3.3 \%)$ \\
\hline CAD & $7(16.7 \%)$ & $0(0 \%)$ & $4(13.3 \%)$ \\
\hline DCM & $1(2.4 \%)$ & $2(4.3 \%)$ & $0(0 \%)$ \\
\hline HHD & $4(9.5 \%)$ & $6(12.8 \%)$ & $6(20.0 \%)$ \\
\hline LA TTE (mm) & $37.5 \pm 3.2$ & $39.6 \pm 4.6$ & $38.9 \pm 4.2$ \\
\hline LVEF normal & $41(97.6 \%)$ & $45(95.6 \%)$ & $24(80.0 \%)$ \\
\hline
\end{tabular}

aincluding beta-blockers as the only antiarrhythmics before ablation procedure. AF Atrial Fibrillation, AADs Antiarrhythmic Drugs, CAD Coronary Artery Disease, DCM Dilatative Cardiomyopathy, HHD Hypertensive Heart Disease, HDMM High Density Mesh Mapper, HDMA High Density Mesh Ablator, LA Left Atrium, LVEF Left Ventricular Ejection Fraction, PV(I) Pulmonary Vein (Isolation), TTE Transthoracic Echocardiography.

\section{Pulmonary Vein Isolation with High Density Mesh Mapper (HDMM)}

The HDMM electrode (Figure 1) and the ablation procedure had been described by our group [4] and Neumann et al [9]. Under fluoroscopy, the transseptal puncture (TSP) was done twice, first for the HDMM, the high-density mapping catheter, and second for the RF ablation catheter. The pre ablation illustration of all PVs by angiography, the carefully mapping and the final position of the HDMM into each PV had also to be completed under ongoing fluoroscopy. Control of the ablation localization was done by fluoroscopy as well as electrophysiologically by the interference of the involved leads in the HDMM.

\section{Pulmonary Vein Isolation with High Density Mesh Ablator (HDMA)}

The HDMA (Figure 2) and the ablation procedure had been described in detail by our group and Steinwender et al. [5,10,11]. Under fluoroscopy, a single TSP and introduction of a long sheath in the LA with selective venography of all PVs was performed. Once, the LA-PV junction was identified, the HDMA was pulled back at the fluoroscopic ostium (set as anatomic ostium). After each circumferential or quadrant RF application (twice, duration of $300 \mathrm{~s}$ ), control of the ablation localization was done by fluoroscopy as well as the evaluation of the amplitude of the PV potential.

\section{Pulmonary Vein Isolation with the Cryobal- loon catheter Arctic Front $\AA$, Cryocath $₫$ TM}

The cryoballoon catheter (Arctic Front ${ }^{\circledR}$, Cryocath ${ }^{\circledR T M}$; Figure 3 ) and its positioning techniques were described in details by Chun et al. [7]. The balloon size was selected accordingly to computed tomography/MRI scan preferring the larger diameter of $28 \mathrm{~mm}$. Under fluoroscopy, the confirmation of good catheter position was done using contrast injection. The position of the cryoballoon during the freeze was documented for all applications. During ablation of the right-sided PVs, the phrenic nerve was continuously stimulated to assess for phrenic nerve palsy. After two freezes $(2 \times 300 \mathrm{~s})$ of each PV, a mapping catheter was inserted into the PV. If this registration showed persistence of the PV potentials, the cryoballoon was introduced again -up to three times - trying to maximize wall contact.

\section{Short- and long-term follow up}

The evaluation of safety was defined from the start of the procedure. Peri-procedural brain MRI to prove embolism was not routinely arranged. AF re- 
currence was counted starting 3 months after the procedure (blanking period). Patients were seen in our clinic every 6 months up to 2 years after ablation. A detailed evaluation of the current medication and the patients' symptoms suggestive for potential AF recurrences was performed. For the evaluation of the rhythm, all patients at intervals of 6 and 12 months received a 7-day Holter recording to take home. Clinical success of the ablation was defined as absence of AF during the prearranged 7-day Holter recording.
Follow-up data after 12 and 24 months were obtained by direct telephone interview.

\section{Statistical Analysis}

Statistical analysis was performed using the SPSS 15.0 (SPSS Inc., Illinois, USA) software package. For the description of the metric variables, the results are expressed as number, mean, standard deviation and extreme (minimum and maximum). The unpaired $t$ test was used to compare mean PD and FT (both in min).

1a:
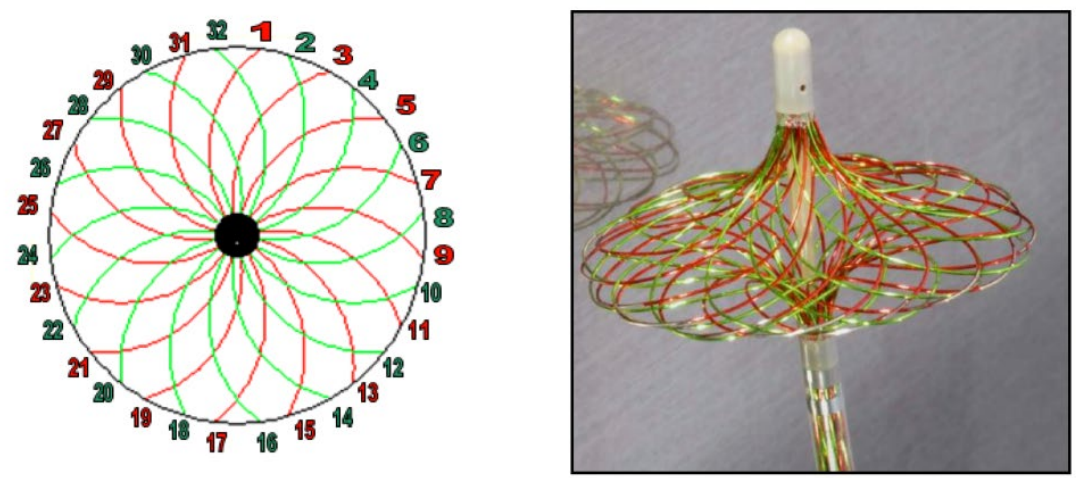

1b:
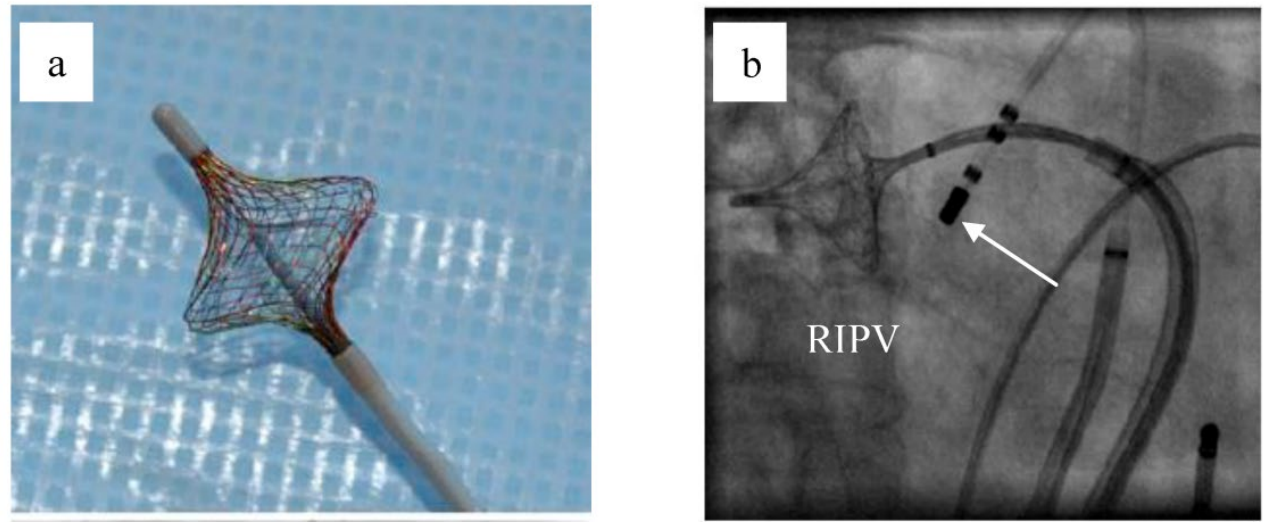

Figure I. Ia: The High Density Mesh Mapper (HDMM) - schematic on the left pannel - is constructed of 32 wires that can be used to record 32 bi-polar electrograms in a clockwise, and/or counter clockwise orientation. Spanned to an umbrella (right pannel), it has a variable range from II-25 mm. Stimulation is possible from any bipole of the Mesh Mapper catheter yielding rapid confirmation of electrical isolation of the pulmonary vein potentials. I b: When fully deployed (a), the mesh disk can be easily positioned at the "true" pulmonary vein ostium (right: right inferior pulmonary vein). The device was only used for high-density mapping. An additional catheter was used for segmental ostial ablation (white arrow in b). Irrigated ablation of each PV was carried out closed but at the exterior part of the HDMM with a target power output of $35 / 40$ Watts and a target temperature of 40 to 45 maximum. 

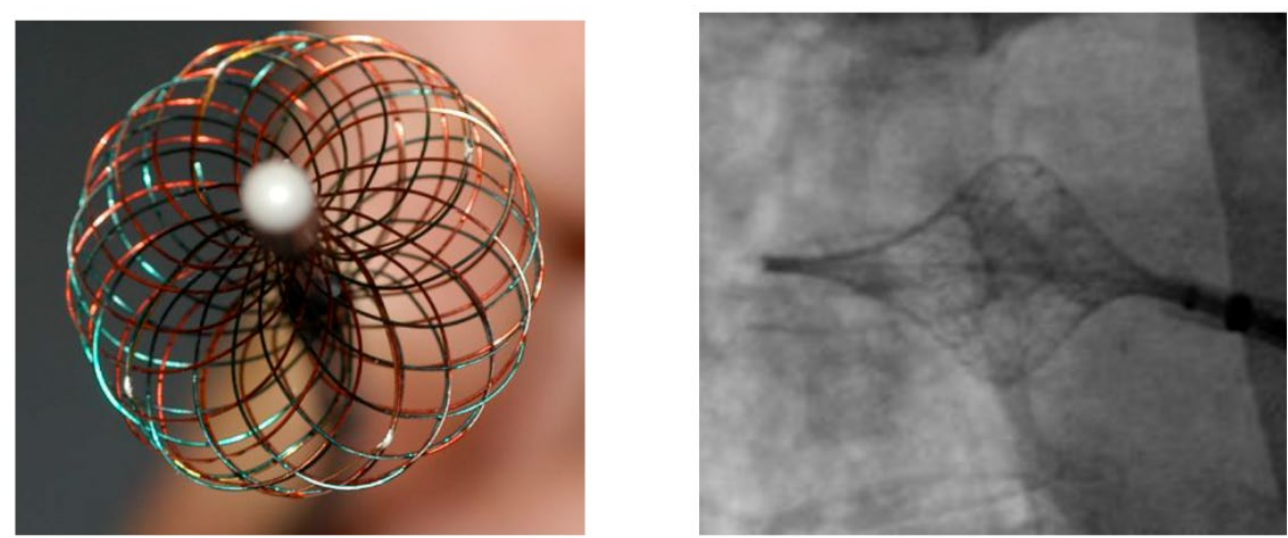

Figure 2: The High Density Mesh Ablator (HDMA) is a non-deflectable $8 \mathrm{~F}$ expandable mesh configured electrode catheter, consisting of twice opposing 18 electrode pairs. In comparison to the High Density Mesh Mapper, the interwoven electrodes of the HDMA allow for complete circumferential mapping and ablation around the pulmonary veins. Systematically, the catheter consists of four quadrants. Four temperature sensors are placed at the edge of each quadrant, providing continuous temperature sensing during the ablation procedure. In conjunction with the 3D mesh configuration of the catheter itself, the HDMA is tagged by three fluoro-markers to improve navigation under fluoroscopy, allowing complete circumferential mapping and ablation around the pulmonary veins by staggered recording configuration.

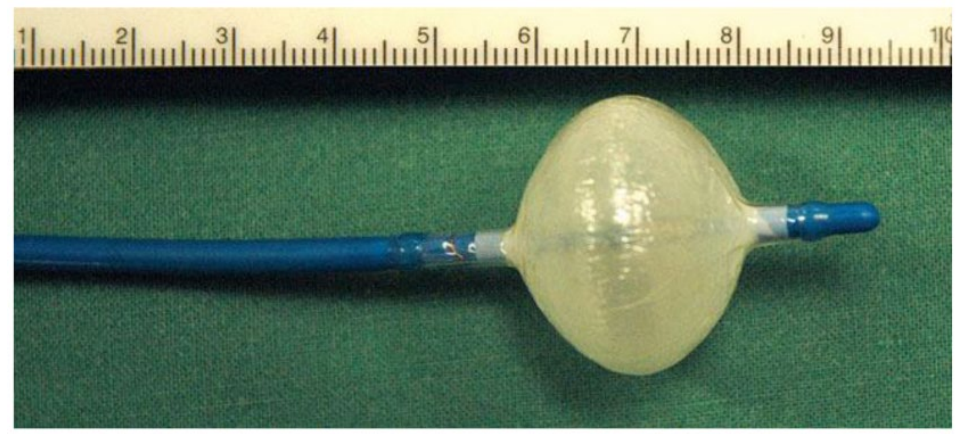

Figure 3: Distal end of a $23 \mathrm{~mm}, 10.5 \mathrm{~F}$, double lumen cryoballoon catheter (Cryocath $®$ ) after inflation.

\section{Results}

\section{Study population}

We studied 119 patients, 75 male $(63.0 \%)$ and 44 female $(37.0 \%)$, mean age $59.4 \pm 10.4$ years). Out of 119 patients, 42 patients were treated with the HDMM $(35.3 \%), 47$ with the HDMA (39.5\%) and 30 with the cryoballoon Arctic Front ${ }^{\circledR}(25.2 \%) .8$ patients were found with a left common ostium (LCO). Table 1 shows the baseline characteristics of 119 patients in the HDMM, HDMA and cryoballoon group.

\section{Procedural data with the HDMM}

Ablation was performed with a mean of $17.4 \pm$ 9.4 RF applications for each application. Concerning the whole study group, the endpoint (the entry block to the vein) could be achieved in $95.5 \%$ of the PVs investigated. Acute success rate were: $98.6 \%$ for LSPV, 95.9\% for LIPV, $98.6 \%$ for RSPV, and $88.9 \%$ for RIPV.
After ablation, five patients $(11.9 \%)$ had a mild, hemodynamic non relevant pericardial effusion.

\section{Procedural data with the HDMA}

Ablation was performed with a mean of $3.15 \pm$ 1.3 RF applications for a mean of $686.7 \pm 237.4$ seconds for each application. Complete PVI as entrance conduction block was obtained in $94.2 \%$ of all PV ablated. Acute success rate were: $97.3 \%$ for LSPV, $97.3 \%$ for LIPV, $97.3 \%$ for RSPV, and $88.1 \%$ for RIPV. After ablation, two patients $(4.3 \%)$ had a mild, hemodynamic non relevant pericardial effusion. None of the patients died due to severe complications, or suffered a hemodynamic relevant pericardial effusion and/or stroke.

\section{Procedural data with Cryoballoon catheter Arctic Front ${ }^{\circledR}$, Cryocath $\AA^{T M}$}

Ablation was performed in 9 patients with the small $(23 \mathrm{~mm})$ and in 21 patients with the big $(28 \mathrm{~mm})$ 
balloon with a mean number of $2.3 \pm 0.6$ freezes per $\mathrm{PV}$ in $565.7 \pm 160.4$ minutes. The mean temperatures were $-55.7 \pm 9.4^{\circ} \mathrm{C}$ for the LSPV, $-50.8 \pm 12.5^{\circ} \mathrm{C}$ for the LIPV, $-53.1 \pm 8.5^{\circ} \mathrm{C}$ for the RSPV and $-46.7 \pm 13.0^{\circ} \mathrm{C}$ for the RIPV. More cryothermal energy applications (3.7 \pm $0.5)$, and longer total freezing time $(800.0 \pm 374.2 \mathrm{~min})$ were required to isolate the LCOs, compared to the LIPV $(2.1 \pm 0.5$ applications and $685.4 \pm 232.1 \mathrm{~min}$, respectively, $p<0.01)$. Complete PVI was achieved in 29 of the 30 LSPVs $(96.7 \%$, including 2 of 3 LCOs), 26 of the 28 LIPVs (95.9\%), 27 of the 30 RSPVs (90.0\%) and 26 of the 30 RIPVs (86.7\%) of the PVs targeted. To eliminate residual PV potentials, we shifted to conventional catheter ablation in two patients. Temporary phrenic nerve palsies was observed in three patients of the cryoballoon group.

\section{Procedure duration and fluoroscopy time}

Figure 4 shows the box plots of the PD and FT in all patients examined. The HDMM group showed significant longer PD than the HDMA group (231.7 \pm $46.3 \mathrm{~min}$ versus $172.5 \pm 42.6 \mathrm{~min}, \mathrm{p}<0.005)$, the HDMA group longer PD than the cryoballoon group (172.5 $\pm 42.6 \mathrm{~min}$ versus $159.5 \pm 36.7 \mathrm{~min}$, without statistical significance). The HDMM group showed significant longer FT than the HDMA group $(70.6 \pm 18.2$ min versus $33.2 \pm 10.2 \mathrm{~min}, \mathrm{p}<0.005)$, the HDMA group shorter FT than the cryoballoon group (33.2 \pm $10.2 \mathrm{~min}$ versus $34.1 \pm 10.7 \mathrm{~min}$, without statistical significance).

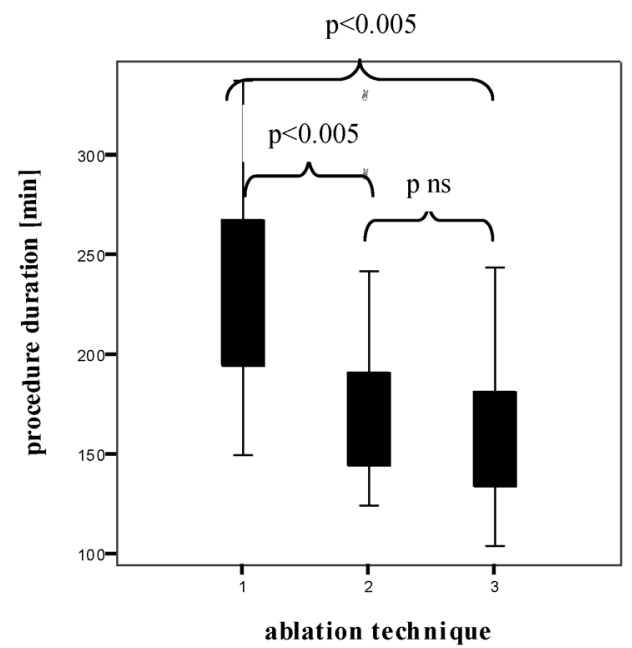

\section{Learning curves}

The learning curve consisted of three unique phases: phase 1 (the initial 10 cases of each ablation tool) represented the initial learning curve, phase 2 (the middle 10 cases), and phase 3 (the subsequent cases). The phase 2 plateau represented increased competence with the technology. When we compared the 30 first procedures in groups of 10 in the HDMM and HDMA group, PD and FT fell significantly $(\mathrm{p}<$ 0.005) in the HDMA group in comparison to the HDMM group (Figure 5a and 5b). In the cryoballoon group, there was a steep learning curve with a steady state after the first 10 procedures. The PD and FT of the first 10 to the following 20 patients decreased significantly from 189.5 and 36.9 to 138.1 and $27.4 \mathrm{~min}$ respectively ( $\mathrm{p}$ values 0.005 and 0.05 respectively).

\section{Follow-up at $\mathbf{6}$ to $\mathbf{2 4}$ months}

Concerning all HDMM patients, $86.1 \%$ had improved clinically, whereas $72.2 \%$ reported to be free of arrhythmias in Holter recordings in a 6 months follow-up. The catheter ablation of AF with HDMA at 12-month interval led to stable SR in not more than $42.9 \%$. In both groups, MRI did not reveal PV stenosis in any patient. Concerning the cryoballoon group, four patients were lost to follow up; $73.1 \%$ had improved and were free of arrhythmias in 6 and 12 month follow-up. After two years, $45 \%$ of these patients were without oral anticoagulants.

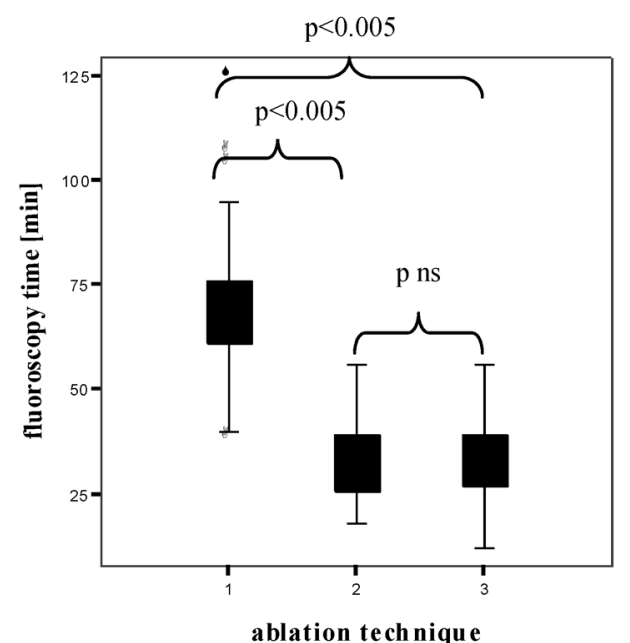

ablation tech nique

Figure 4: Box plots of the procedure duration and the fluoroscopy time (both in min) I High Density Mesh Mapper, 2 High Density Mesh Ablator, 3 Arctic Front $®$ Cryoballoon. 
5 a

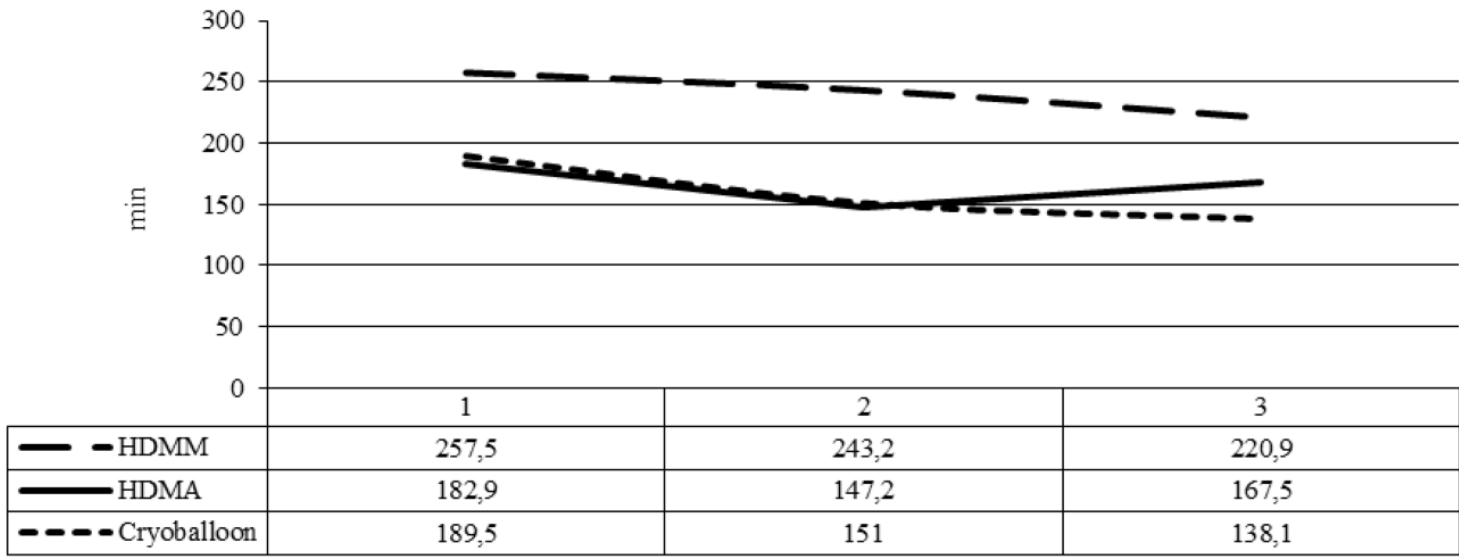

patients

$5 \mathbf{b}$

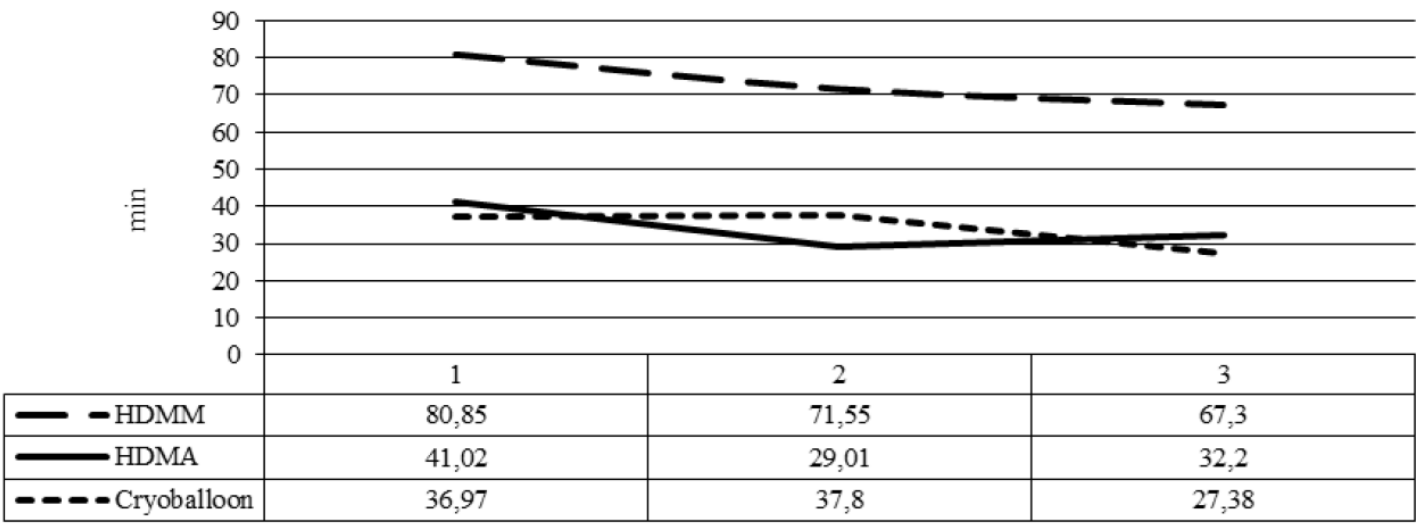

patients

Figure 5. 5a: Procedure duration (minutes) averaged per group of 10 consecutive procedures (I Patients I-I0, 2 Patients II -20, 3 Patients 2I-30). 5b: Fluoroscopy times (minutes) averaged per group of I0 consecutive procedures (I Patients I-I0, 2 Patients II-20, 3 Patients $21-30)$.

\section{Discussion}

This study aimed to compare the procedural characteristics of three fluoroscopy guided ablation tools in AF and their efficacy in long-term follow up. Despite a significant reduction of procedural and fluoroscopy times in the HDMA compared with the HDMM group, the study findings suggest that although the HDMA facilitate the procedure, the efficacy is insufficient in long-term follow up. The use of cryoballoon technique had the shortest learning curve, resulted in the shortest procedure duration and in a further reduction in fluoroscopy exposure compared with HDMM and HDMA. Moreover, in this study, the improvements in the fluoroscopy process were achieved without reduction in long-term clinical outcomes. The safety was acceptable in all devices.

\section{Potential advantages and potential disad- vantages of the different ablation tools}

\section{The HDMM and HDMA catheter}

The safety and feasibility of the flexible, variable-sized device designed to accommodate diverse 
veno-atrial anatomy was assessed by Arruda [13]. Our group described, that the majority of the PVs could be examined despite the relatively stiff mapping tool [4]. The mesh offers beside excellent electrophysiological control a very good $3 \mathrm{~d}$ orientation because of the basket configuration, which allows an easy anatomical understanding, even at the complex LA-PV junction. The radiation field during fluoroscopy can be minimized to the mesh itself and the nearest surrounding.

The PVI with the HDMM requires two TSPs in a single procedure for the introduction of the mapping catheter itself and an additional catheter for RF delivery. Ablating at the atrial border of the LA-PV junction makes this approach not only to a pure ostial ablation approach, but can be interpreted as a >supra-ostial< ablation approach including major fractions of the atrial tissue. Ablating deep in the atrium with point by point ablation around the HDMM was time consuming. The PD for patients treated with the HDMM was therefore the longest in our study with $231.7 \pm 46.3 \mathrm{~min}$, our FT the highest with $70.6 \pm 18.2 \mathrm{~min}$. In the literature, there are FTs of $42 \pm 20$ min with an average of 5 to 7 ablations [14].

The catheter design of the HDMA combining high-density circumferential mapping and direct RF energy delivery in a single unit has been developed for a safer and notably faster procedure mainly due to a single transseptal access. For that reason, the PD with HDMA could significantly to be reduced in comparison to the HDMM. The technology of the HDMA provided also the possibility of energy delivery in a circular and quadrant mode of the HDMA electrode. In comparison with the HDMM ablation procedure, the ablation with the HDMA is a more PV-ostium-related technique with all the well-known disadvantages previously described [12]. In order to overcome the limitations associated with RF energy via small elements, RF was delivered in a pulsed, temperature-control mode. This form of RF delivery prevents tissue overheating, impedance rise, and char formation. As discussed by our own group [14], the advantages of the catheter may translate into a shorter PD but maybe lead to poor long-term results due to the fact that effective transmural lesions cannot be achieved with the electrodes.

The potential risks of placement of basket catheters in the PVs should be emphasized. Although we encountered no difficulties in the cases reported here, we envision a potential risk of thromboembolism. To minimize the risk, we maintained full anticoagulation throughout deployment of the catheter, with regular checks of the activating clotting time.

\section{Cryoballoon catheter Arctic Front $₫$, Cry- ocath $\AA^{\mathrm{TM}}$}

Using a simple over-the-wire technique in conjunction with a steerable sheath, the balloon can be easily navigated to each PV ostium. With regard to safety aspects, cryothermal energy may have advantages over other energy sources since both human and experimental animal data have demonstrated that the risk for PV stenosis [15], atrio-oesophageal fistulae [16], and thrombus formation [17] is extremely low. Cryothermal energy balloon-based ablation is designed to achieve complete circular lesions around the PVs with a single application of energy, independent of the individual PV anatomy. This ablation technology facilitates PVI and improves the safety of the procedure. The study by Chun et al. [7] was achieved by two investigators with no prior experience with the cryoballoon technology. This underscores the fact that cryoballoon ablation of PVs is a simple and straightforward procedure with a fast learning curve. In our study, the cryoballoon technique yield to decreased PD and FT (Figure 5a and b). The main disadvantage was the fact that if a PV could not be completely isolated with the first freeze, several freezes were necessary with prolongation of PD and FT.

From a procedural point of view, the percentage of PVs that could be isolated using the HDMM was $95.5 \%$, using the HDMA $94.2 \%$ and the cryoballoon $92.3 \%$ including 2 patients with touch up technique. The latter result was equal compared to the study by Van Belle et al., but lower than in the studies by Chun et al. and Klein et al. who were able to isolate $98 \%$ and $95 \%$ of all PVs, respectively $[6,7,18]$. In our series, the PD was smaller compared to what large centres have published (168.6 $\pm 37.8 \mathrm{~min})$. Chun et al. reported a median procedure time of $220 \mathrm{~min}$ using the "single big cryoballoon" technique. They did not require a shift to conventional ablation catheter in any of their patients in order to achieve a very high PV isolation rate of $98 \%$. In order to achieve that, they used up to 9 applications of cryoenergy $(45 \mathrm{~min})$ on a single PV, and up to 13 applications $(65 \mathrm{~min})$ in case of the presence of a LCO. In contrast, we never exceeded more than 4 applications of cryoenergy for treating a single PV. Van Belle et al. reported similar PDs when using only the balloon $(211 \pm 108 \mathrm{~min})$ and also had somewhat longer PDs when switching to a conventional catheter $(261 \pm 83 \mathrm{~min})$. In the study by Neumann et al., the PD was 170 min [8]. They were able to isolate $97 \%$ of all PVs using one or two balloons, or a balloon in combination with a conventional catheter. 


\section{Paradigm shift or not}

Our study reflects well the change in the performance of PVI in patients with AF within the ruling theory of Haissaguerre [19]. The foregoing decade focussed on either time consuming electrophysiological guided direct ablation of each PV potential $[20,21,22]$ or on a 3D electro-anatomical guided approach to electrically isolate the PVs by creating hemispheric or circumferential lesions at the PV ostium $[23,24,25,26]$. Limitations of the approaches included smooth learning curves, the need for more than one transseptal catheter, prolonged PD, and high risk of complications.

Nowadays, the main goal seems to be to investigate a higher number of patients. The attention is turning towards a purely anatomical approach with the intent to find an alternative method that should be as effective but more easily with shorter PDs and reduced complexity. These intentions lead to 'single shot' PVIs allowing ablations to be performed by operators with little prior experience. The promising results with the cryoballoon technique may offer a perspective in the treatment of $\mathrm{AF}$ [27].

As an inevitable consequence, the question arises if "efficacy" or "time" is everything. The possibility to prevent AF recurrence is a strong indicator of quality of ablation but PD and FT should also be included.

\section{Conclusion}

The study was designed to compare the safety and efficacy of different fluoroscopy guided ablation tools performing PVI in patients with PAF or persAF. Our retrospective analysis of the PD and FT represents characteristic stages of the learning curve for PVI procedures. In comparison to HDMM and HDMA, the single big cryoballoon technology has an acceptable learning curve and clearly has the potential to make PVI simple, fast, and safe. The HDMM is comparable in long-term outcome, but associated with longer PD and FT.

\section{Limitations of the present study}

This study represents a retrospective analysis of data on AF ablation at our single centre and was carried out in a relatively small cohort, comprising of patients with PAF and persAF. We did not compare our fluoroscopy guided ablation tools with one of the several nonfluoroscopic mapping systems, nowadays used to guide catheter ablation of AF in creating $3 \mathrm{D}$ electroanatomic maps of the left atrium, because these ablation tools were not the primary focus of this analysis. Using HDMM, HDMA and the cryoballoon alone, the PD of the available ablation tools intended for PVI is only partly comparable because of their individual design with a need or not for additional catheters. Observational studies like this can provide additional information, since they are fully representative of everyday clinical practice, in which several factors underestimated in prospective trials, such as the different experience of the involved centers, may play a role. For this reason, observational studies will be of particular importance in monitoring and guiding the incorporation of this therapeutic procedure into clinical practice.

\section{Abbreviations}

$\begin{array}{ll}\text { AF: } & \text { Atrial Fibrillation; } \\ \text { HDMA: } & \text { High Density Mesh Ablator; } \\ \text { HDMM: } & \text { High Density Mesh Mapper; } \\ \text { FT: } & \text { Fluoroscopy Time; } \\ \text { LA: } & \text { Left Atrium; } \\ \text { LIPV: } & \text { Left Inferior Pulmonary Vein; } \\ \text { LSPV: } & \text { Left Superior Pulmonary Vein; } \\ \text { PAF: } & \text { Paroxysmal Atrial Fibrillation; } \\ \text { PD: } & \text { Procedure Duration; } \\ \text { PersAF: } & \text { Persistent atrial fibrillation; } \\ \text { PV: } & \text { Pulmonary Vein; } \\ \text { PVI: } & \text { Pulmonary Vein Isolation; } \\ \text { RF: } & \text { Radio Frequency; } \\ \text { RIPV: } & \text { Right Inferior Pulmonary Vein; } \\ \text { RSPV: } & \text { Right Superior Pulmonary Vein; } \\ \text { TSP: } & \text { Transseptal Puncture. }\end{array}$

\section{Competing Interests}

The authors have declared that no competing interest exists.

\section{References}

[1] European Heart Rhythm Association; European Association for Cardio-Thoracic Surgery, Camm AJ, Kirchhof P, Lip GY, Schotten U, Savelieva I, Ernst S, Van Gelder IC, Al-Attar N, Hindricks G, Prendergast B, Heidbuchel H, Alfieri O, Angelini A, Atar D, Colonna P, De Caterina R, De Sutter I, Goette A, Gorenek B, Heldal M, Hohloser SH, Kolh P, Le Heuzey JY, Ponikowski P, Rutten FH. Guidelines for the management of atrial fibrillation: the Task Force for the Management of Atrial Fibrillation of the European Society of Cardiology (ESC). Eur Heart J. Oct 2010;31(19): 2369-429.

[2] Wright M, Haïssaguerre M, Knecht S, Matsuo S, O'Neill MD, Nault I et al. State of the art: catheter ablation of atrial fibrillation. J Cardiovasc Electrophysiol. 2008; 19: 583-592.

[3] Dewire, J., Calkins, H. State-of-the-art and emerging technologies for atrial fibrillation ablation. Nat Rev Cardiol. 2010 Mar;7(3): 129-38.

[4] Meissner A, van Bracht M, Schrage MO, Christ M, Trappe HJ, Maagh P et al. Segmental pulmonary vein isolation in atrial fibrillation: new insights from the high density mesh mapper technique in an electrophysiologically guided approach. J Interv Card Electrophysiol. 2009 Sep;25(3): 183-92.

[5] Meissner A, Plehn G, van Bracht M, Schrage MO, Christ M, Maagh P et al. First Experiences for Pulmonary Vein Isolation with the High-Density Mesh Ablator (HDMA): A Novel Mesh Electrode Catheter for Both Mapping and Radiofrequency Delivery in a Single Unit. J Cardiovasc Electrophysiol. 2009 Apr; 20(4): 359-66.

[6] Van Belle Y, Janse P, Rivero-Ayerza MJ, Thornton AS, Jessurun ER, Theuns D et al. Pulmonary vein isolation using an occluding cryoballoon 
for circumferential ablation: feasibility, complications, and shortterm outcome. Eur Heart J. 2007 Sep; 28(18): 2231-7.

[7] Chun KR, Schmidt B, Metzner A, Tilz R, Zerm T, Köster I et al. The 'single big cryoballoon' technique for acute pulmonary vein isolation in patients with paroxysmal atrial fibrillation: a prospective observational single centre study. Eur Heart J. 2009 Mar;30(6): 636.

[8] Neumann T, Vogt J, Schumacher B, Dorszewski A, Kuniss M, Neuser H et al. Circumferential Pulmonary Vein Isolation With the Cryoballoon Technique Results From a Prospective 3-Center Study. J Am Coll Cardiol. 2008 Jul 22; 52(4): 273-8.

[9] Neumann T, Kuniss M, Erkapic D, Zaltsberg S, Berkowitsch A, Pajitnev $\mathrm{D}$ et al. Acute and long-term results of PVI at antrum using a novel high-density mapping catheter without help of 3D electro-anatomic mapping in patients with paroxysmal and chronic atrial fibrillation. J Interv Card Electrophysiol. 2010 Mar; 27(2): 101-8.

[10] Steinwender C, Hoenig S, Leisch F, Hofmann R. Acute Results of Pulmonary Vein Isolation in Patients with Paroxysmal Atrial Fibrillation Using a Single Mesh Catheter. J Cardiovasc Electrophysiol. 2009 Feb; 20(2): 147-52.

[11] Steinwender C, Hoenig S, Leisch F, Hofmann R. One-year follow-up after pulmonary vein isolation using a single mesh catheter in patients with paroxysmal atrial fibrillation. Heart Rhythm 2010; 7: 333-339.

[12] Maagh P, van Bracht M, Butz T, Trappe HJ, Meissner A. Eighteen months follow-up of the clinical efficacy of the high density mesh ablator (HDMA) in patients with atrial fibrillation after pulmonary vein isolation. J Interv Card Electrophysiol. 2010 Oct; 29(1): 43-52.

[13] Arruda MS, He DS, Friedman P, Nakagawa H, Bruce C, Azegami K et al. A novel Mesh electrode catheter for mapping and radiofrequency delivery at the left atrium-pulmonary vein junction: A simplified approach to pulmonary vein isolation. J Cardiovasc Electrophysiol 2007; 18: 206-211.

[14] Chen SA, Hsieh MH, Tai CT, Tsai CF, Prakash VS, Yu WC et al. Initiation of atrial fibrillation by ectopic beats originating from the pulmonary veins: Electrophysiological characteristics, pharmacological responses, and effects of radiofrequency ablation. Circulation 1999; 100: 1879-1886.

[15] Tse HF, Reek S, Timmermans C, Lee KL, Geller JC, Rodriguez LM et al. Pulmonary vein isolation using transvenous catheter cryoablation for treatment of atrial fibrillation without risk of pulmonary vein stenosis. J Am Coll Cardiol 2003;42: 752-758.

[16] Ripley KL, Gage AA, Olsen DB, Van Vleet JF, Lau CP, Tse HF. Time course of esophageal lesions after catheter ablation with cryothermal and radiofrequency ablation: implication for atrio-esophageal fistula formation after catheter ablation for atrial fibrillation. J Cardiovasc electrophysiol 2007;18: 642-646.

[17] Khairy P, Chauvet P, Lehmann J, Lambert J, Macle L, Tanguay JF et al. Lower incidence of thrombus formation with cryoenergy versus radiofrequency catheter ablation. Circulation 2003;107: 2045-2050.

[18] Klein G, Oswald H, Gardiwal A, Lüsebrink U, Lissel C, Yu H et al. Efficacy of pulmonary vein isolation by cryoballoon ablation in patients with paroxysmal atrial fibrillation. Heart Rhythm 2008; 5: 802-6.

[19] Haissaguerre M, Jais P, Shah DC, Takahashi A, Hocini M, Quiniou G et al. Spontaneous initiation of atrial fibrillation by ectopic beats originating in the pulmonary veins. N Engl J Med 1998; 339: 659-666.

[20] Haïssaguerre M, Shah DC, Jaïs P, Hocini M, Yamane T, Deisenhofer I et al. Electrophysiological breakthroughs from the left atrium to the pulmonary veins. Circulation. 2000; 102: 2463-2465.

[21] Oral H, Knight BP, Tada H, Ozaydin M, Chugh A, Hassan S et al. Pulmonary vein isolation for paroxysmal and persistent atrial fibrillation. Circulation. 2002; 105: 1077-1081.

[22] Takahashi A, Iesaka Y, Takahashi Y, Takahashi R, Kobayashi K, Takagi K et al. Electrical connections between pulmonary veins: implications for ostial ablation of pulmonary veins in patients with atrial fibrillation. Circulation. 2002;105: 2998-3003.

[23] Pappone C, Oreto G, Rosanio S, Vicedomini G, Tocchi M, Gugliotta F et al. Atrial electroanatomical remodeling after circumferential radiofrequency pulmonary vein isolation: efficacy of an anatomic approach in a large cohort of patients with atrial fibrillation. Circulation. 2001;104: 2539-2544.

[24] Oral H, Scharf C, Chugh A, Hall B, Cheung P, Good E et al. Catheter ablation for paroxysmal atrial fibrillation: segmental pulmonary vein ostial ablation versus left atrial ablation. Circulation. 2003;108: 2355-2360.

[25] Ernst S, Ouyang F, Löber F, Antz M, Kuck KH et al. Catheter-induced linear lesions in the left atrium in patients with atrial fibrillation. J Am Coll Cardiol. 2003;42: 1271-1282.

[26] Pappone C, Santinelli V, Manguso F, Vicedomini G, Gugliotta F, Augello $\mathrm{G}$ et al. Pulmonary vein denervation enhances long-term benefit after circumferential ablation for paroxysmal atrial fibrillation. Circulation. 2004;109: 327-334.

[27] Hofmann R, Hönig S, Leisch F, Steinwender C. Pulmonary vein isolation with Mesh Ablator versus cryoballoon catheters: 6-month outcomes. J Interv Card Electrophysiol. 2010 Dec;29(3): 179-85. 